\title{
The Operational Effects of Implementing Electronic Provider Documentation in the Emergency Department
}

\section{Citation}

Feblowitz, Joshua C. 2015. The Operational Effects of Implementing Electronic Provider Documentation in the Emergency Department. Doctoral dissertation, Harvard Medical School.

\section{Permanent link}

http://nrs.harvard.edu/urn-3:HUL.InstRepos:17295877

\section{Terms of Use}

This article was downloaded from Harvard University's DASH repository, and is made available under the terms and conditions applicable to Other Posted Material, as set forth at http:// nrs.harvard.edu/urn-3:HUL.InstRepos:dash.current.terms-of-use\#LAA

\section{Share Your Story}

The Harvard community has made this article openly available.

Please share how this access benefits you. Submit a story.

\section{Accessibility}




\section{Table of Contents}

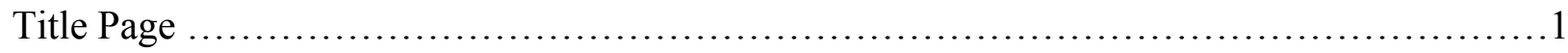

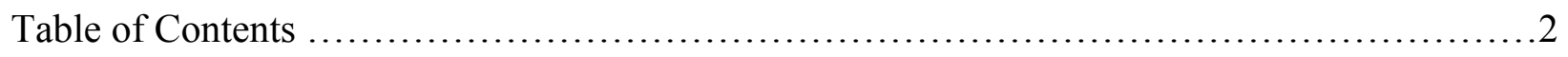

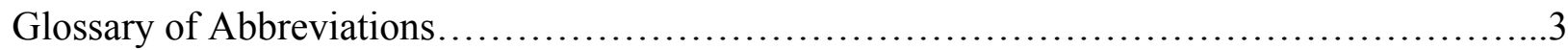

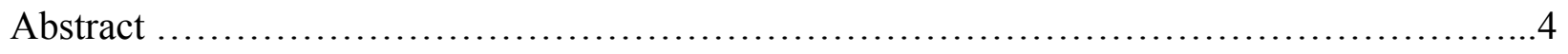

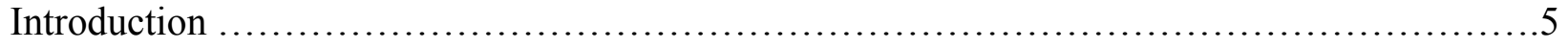

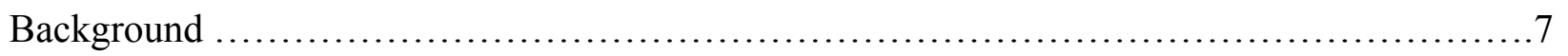

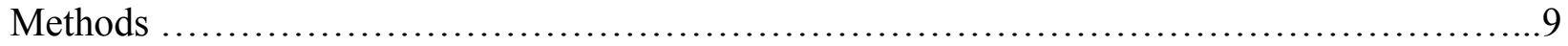

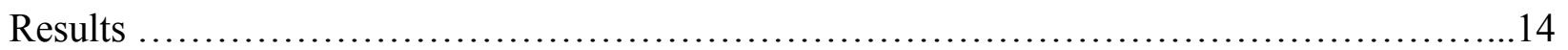

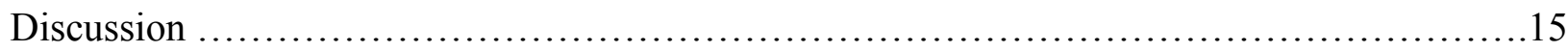

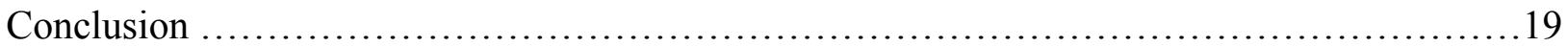

Suggestions for Future Work .................................................. 19

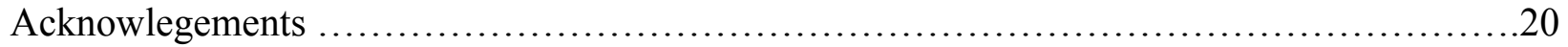

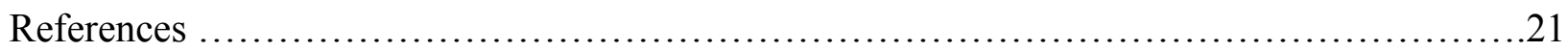

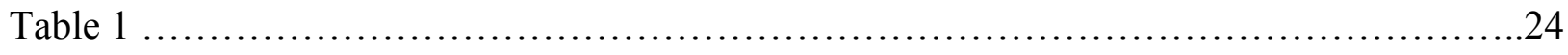

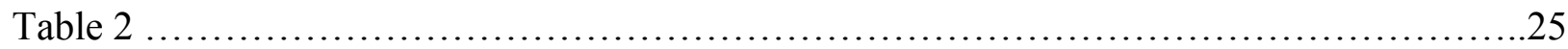

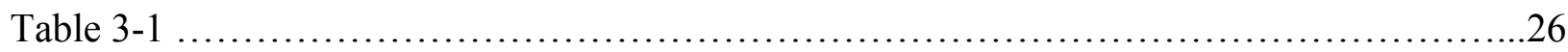

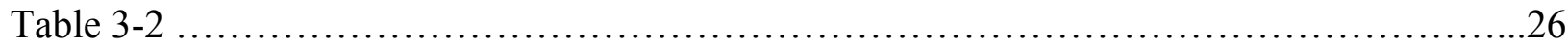

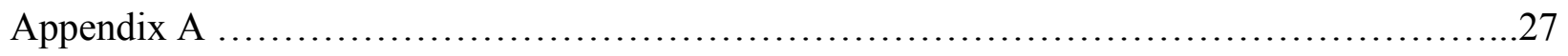

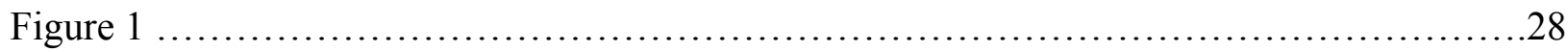

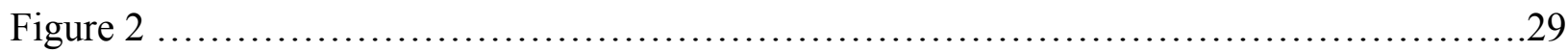




\section{Glossary of Abbreviations}

In order of appearance in the text of this report:

EHR - electronic health record

ED - emergency department

eDoc - custom electronic documentation system used at Brigham \& Women's Hospital

$\mathrm{CPOE}$ - computerized provider order entry

e-documentation - electronic provider documentation (generally)

LOS - length of stay

LOSa - length of stay for admitted patients

LOSd - length of stay for discharge patients

TTD - time to disposition for admitted patients

CEM - coarsened exact matching

HITECH - Health Information Technology of Economic and Clinical Health

BWH - Brigham \& Women's Hospital

PA - physician's assistant

IRB - institutional review board

MRN - medical record number

ESI - emergency severity index

DOB - date of birth

OBS - observation admission

SATT - sample average treatment effect on the treated 


\section{Abstract}

Background: EHR implementation may improve care quality in the ED. At our institution, we implemented a custom e-documentation system (eDoc) to replace paper documentation. No studies to date have characterized the effect of implementing e-documentation in the ED.

Objective: To characterize the operational effects of implementing eDoc in our ED.

Methods: We performed a retrospective analysis of data for 1-year periods before and after implementation. We used regression modeling and CEM to identify significant differences in outcome variables.

Results: During the pre-implementation period, LOS was 4.29 hours, LOSa was 6.47 hours and LOSd was 3.49 hours; after implementation, LOS for these groups were 4.43 hours, 6.66 hours, and 3.52 hours. TTD was 3.00 hours before and 3.03 hours after implementation. Using regression analysis, there were no differences in outcome variables at 8 weeks; at one year, there were differences in LOS and LOSd patients of $\Delta+0.10$ hours and $\Delta+0.08$ hours. CEM analysis demonstrated a change of $\Delta+0.15$ hours and $\Delta+0.17$ hours for LOS and LOSd.

Conclusions: In our study, implementation of e-documentation was associated with significant increases in LOS and LOSd. Though this increase may appear small, this additional time required for e-documentation has the potential to impact ED efficiency. 


\section{Introduction}

In recent years, national policy initiatives and significant government funding have encouraged the design and implementation of electronic health record systems (EHRs) across the country with the goal of improving the quality of patient care and reducing healthcare costs. Through the Health Information Technology for Economic and Clinical Health (HITECH) Act of 2009, the federal government has invested substantial resources to encourage the adoption of EHRs by hospitals and physicians and the use of EHRs has expanded rapidly in recent years under this program (1-4).

The HITECH Act - a component of the American Recovery and Reinvestment Act of 2009 - made \$19.2 billion available to encourage adoption of EHRs in the form of incentive payments to providers and hospitals (5). As part of this program, incentive payments of up to $\$ 44,000$ through Medicare or $\$ 63,750$ through Medicaid are awarded for adopting an EHR. In order to receive these incentive payments, providers and hospitals must i) use a certified EHR technology, ii) submit clinical quality measures iii) demonstrate "meaningful use" of the EHR by meeting several objectives for recording patient data electronically (6). Given the difficulty of implementing EHRs, the meaningful use program created three separate stages spanning five years, so hospitals and providers could gradually increase their EHR functionality over time.

The most recent meaningful use rules outline the requirements for receiving incentive payments under Stage 2 of the EHR incentive program. For the first time, these requirements include an objective to record patient notes electronically (7). Menu objective 2 of Stage 2 rules require that hospitals record an electronic note for 30 percent of unique patients admitted to the emergency department or hospital. Numerous other requirements for use of the EHR are part of the meaningful use goals, such as recording patient demographics, gender, age, and smoking status electronically, utilizing computerized provider order entry (CPOE), recording vitals, displaying laboratory data, and implementing clinical decision support rules (8). Criteria for meeting meaningful use will continue to become more stringent over time and providers and hospitals that do not meet these goals will not receive incentive payments and may ultimately be subject to reimbursement penalties.

Implementation of EHRs has been shown to improve efficiency and patient safety in the ED (9-13) and, currently, implementation of ED information systems remains has been expanding rapidly (14). A recent CDC report found that the percentage of EDs with EHRs 
increased from $46 \%$ in 2006 to $84 \%$ in 2011 and the proportion of EDs with at least a basic EHR tripled to $54 \%$. However, according to the CDC report and another study by Landman et al (2010) only a small minority of EDs have fully functional systems that meet meaningful use objectives $(14,15)$

Furthermore, in some studies, the implementation of these systems has had either a transient or sustained negative impact on departmental efficiency, increasing documentation time and length of stay significantly (16-19). In a 2013 study by Ward et al (20), implementation of an EHR was found to be associated with a transient increase in length of stay and decrease in patient satisfaction scores at a single center but this effect was not sustained over time. In addition, in this study, rates of laboratory testing, medication administration, imaging and EKG ordering showed a sustained increase after implementation of the EHR. In another recent large study of 23 diverse emergency departments (21), implementation of an EHR was not found to be associated with a significant change in multiple measures of operational performance, including arrival-to-provider time, admitted length of stay, discharged length of stay, overall length of stay, proportion of patients leaving prior to completion of treatment, return visits, patient satisfaction or provider efficiency. In a small study of ED physician charting time (18), use of an EHR at a single center was found to be associated with longer time to completion of each chart (as measured by stopwatch) but this study did not examine the impact of this change on overall operational performance of the department.

In summary, existing literature on the impact of implementing EHRs in the ED setting have demonstrated a variety of findings, sometimes inconsistent between separate studies. Furthermore, no study to date has examined the isolated effect of implementing electronic documentation, or "charting", in the ED setting. Our study aims to add to what is known about the effect of implementing electronic systems in the ED, and specifically to characterize the isolated effect of implementing e-documentation, separating this from other functions of an EHR that could have an effect on departmental efficiency. Given the substantial regulatory and financial impetus to encourage the use of EHRs coupled with the importance of efficient ED operations to ensure timely and safe care, it is especially critical to improve our understanding of the impact of these systems and how they can be most effectively deployed in the ED setting. 


\section{Background}

The widespread implementation of electronic health records (EHRs) - which incorporate electronic patient tracking, computerized provider order entry (CPOE), and electronic provider documentation (e-documentation), among other features - has the potential to improve care quality in the emergency department (ED) setting. In the ED - an environment that depends on rapid and highly efficient patient evaluation - the integration of these systems presents both an opportunity to improve care and a unique challenge to ensure continued operational efficiency during and after implementation. Although EHRs can be used in a variety of ways to improve care quality and safety, one of the core functions of an EHR is the ability to document patient encounters electronically, thus eliminating the need for handwritten, hardcopy documentation that is less legible, searchable and accessible. ED physicians and other clinician staff, like many healthcare professionals, devote a substantial amount of time each day to documentation of patient encounters as mandated by regulatory and billing requirements. Electronic charting - and an EHR more broadly - has the potential to improve the accuracy and completeness of patients records, provide quicker access to this information, to improve care coordination and to share information more effectively between providers and patients (22).

Recently, our ED (Brigham and Women's Hospital, BWH, Boston, MA) underwent a three-year custom software development project to transition clinicians from paper-based to edocumentation. The transition to this custom electronic documentation system (henceforth referred to as eDoc) provided an opportunity to study the effect of implementing such computerized systems in the unique ecosystem of the ED. Furthermore, understanding the impact of this new system on core ED quality and operational metrics, such as length of stay, could contribute to the more effective implementation of these systems in the future. Anecdotally, our clinicians reported that e-documentation required additional time compared with paper documentation and initially there was a significant adjustment period as staff got used to the new system. We sought to conduct more rigorously evaluation of this general impression by examining key operational metrics of our ED before and after implementation.

Two common features of EHRs - E-documentation and computerized provider order entry (CPOE) - are frequently implemented together, making it difficult to assess the independent impact of each function. However, at our institution, we implemented eDoc to replace paper-based documentation in the setting of existing electronic patient tracking and 
CPOE. Thus, in this study we were able to specifically examine the isolated effect of edocumentation on departmental efficiency.

Prior research (20) has demonstrated transient negative effects on ED efficiency after implementation of EHRs but also show that these effects were not sustained over time. However, no studies to date have characterized the isolated effect of implementing e-documentation in the ED. CPOE likely has a significant independent impact on operational and quality measures in the ED, such as length of stay (13). Thus, the separate implementation of these systems at our institution allowed us to isolate the effect of transitioning to e-documentation, whereas most hospitals implement these systems simultaneously as part of a complete EHR implementation.

\section{Scholarly Question \& Significance}

The goal of this scholarly project is to characterize the short and long-term effects of the implementation of eDoc on core operational performance measures in the BWH ED over a twoyear period.

Our research is guided by the following specific questions:

- Did the implementation of e-documentation in the BWH ED have a significant effect on key operational measures including overall length of stay, length of stay for admitted patients, length of stay for discharged patients and time to disposition for admitted patients when controlling for variation in visit volume, patient acuity and other operational characteristics?

- Did e-documentation have any short-term effect on the above operational metrics during the 8 weeks following implementation?

- Is any impact (positive or negative) of e-documentation sustained at one year after implementation?

Based on prior literature $(17-20,23)$, we initially hypothesized that the implementation of e-documentation may have resulted in transient decreases in performance measures related to ED efficiency (i.e. increased length of stay), but that over a longer period e-documentation had a positive or neutral effect on departmental efficiency. 
The overarching goal of this study was to characterize the impact of implementing eDoc in our high-volume, urban ED on key operational measures. This research helps us to better understand the impact of implementing e-documentation in the ED setting, provides valuable insight into the current quality of care in the ED and will inform future interventions and policy.

\section{Methods}

\section{Setting}

This study consists of a retrospective analysis of previously collected ED operational data for the 43-bed, urban, academic ED of Brigham \& Women's Hospital (BWH), a 793-bed teaching hospital and tertiary care center located in the Longwood Medical Area of Boston, MA. The annual volume of the BWH ED is approximately 60,000 patient visits. As part of ongoing quality control and patient safety measures, the BWH ED collects data on multiple operational and quality statistics on a rolling basis and publishes this data monthly.

Pre-implementation provider documentation in the BWH ED relied primarily on paper records. Before the implementation of eDoc, documentation was completed on paper by residents and PAs. Attendings used a traditional phone dictation system, with subsequent review of the final transcribed file. We custom developed eDoc to work with our existing electronic ED patient tracking system and electronic order entry system. With the implementation of eDoc, residents and PAs now enter documentation electronically. Attending physicians have the option of typing their notes in eDoc or using voice recognition tool, that immediately transcribes speech into text in eDoc (Nuance, Speech Anywhere 360 Direct).

\section{Data Collection}

After IRB approval, we obtained all logged operational and quality data from the BWH ED for the full study period. eDoc was implemented on March 18, 2013 and we collected data for a one-year pre-implementation period from March 18, 2012 to March 17, 2013 and one-year post-implementation period from March 18, 2013 to March 17, 2014. All recorded patient encounters during the designated study period were included in this analysis.

No other major IT implementation projects, system changes or changes to staff coverage occurred during the study period. 


\section{Dataset}

The primary author (JF) was responsible for review, cleaning and analysis of the full dataset and was assisted as needed with statistical analysis by co-author ST.

The complete dataset obtained from the BWH ED included operational ED data for all registered patient visits including patient medical record number (MRN), arrival date and time, bed request date and time for admitted patients, discharge date and time, emergency severity index (ESI; on a scale of 1-5, with 1 reflecting patients with the highest level of acuity), mode of arrival (ambulance or other) and disposition (inpatient admission, ED observation, home or other). In addition, we obtained Press-Ganey patient satisfaction scores for discharged ED patients. However, these data were only available on an aggregate monthly basis and thus were excluded from daily analysis.

Patient MRN was used to query our EHR and collect gender and date of birth for all patients. A subset of patient MRNs $(n=478)$ did not return gender and DOB data with this query and had to be manually reviewed. Once this step was complete, all protected health information were then removed from the dataset and each patient encounter was assigned a unique randomly generated identification number in order to protect patient privacy.

Additional computed variables were then derived from the dataset by primary author JF. Age was derived from date of visit minus patient DOB. Length of stay (LOS) for all patients and boarding time for admitted patients was then derived from individual encounter data. LOS was defined as recorded arrival time minus recorded discharge time. In addition, boarding time for all admitted patients was calculated based on the discharge time minus bed request time. Time to disposition for admitted patients was calculated by subtracting bed request time from arrival time.

Using this complete dataset, primary author (JF) then calculated summary statistics for all days during the pre- and post-implementation periods, which served as the primary dataset for our analysis. Patient data was included in daily totals based on the arrival date to the emergency department (i.e. data for all patient encounters up to 11:59PM arrival time was included in that day's totals even if the patient remained in the ED through the following day or beyond). Daily variables included pre- or post-implementation period ( 0 or 1), month and day of visit, total registered daily visits, total registered visits for the previous day, days with visits in the upper quartile (upper quartile $>177$ patient visits, 0 or 1), days with visits in the lower quartile (lower 
quartile $>153,0$ or 1), mean patient age, count and proportion of female patients, count and proportion of admissions, count and proportion of ED observation admissions, count and proportion of discharges, proportion of patients with ESI of 2 or 1 (highest acuity), weighted mean ESI, count and proportion of patients of each ESI category (1 through 5), count and proportion arriving by ambulance, count and proportion of arrival by mode other than ambulance, mean time to disposition for admitted patients, total patient hours, mean daily length of stay (LOS), median daily LOS, mean LOS for admitted patients, mean LOS for discharged patients, total boarding hours, and mean boarding time for admitted patients. Daily satisfaction survey data was not available and was thus excluded from further analysis.

Binary variables were assigned a value of 0 or 1 (pre-implementation $=0$, postimplementation $=1$; visits $\leq 177=0$, visits $>177=1$; visits $>153=0$, visits $<153=1$ ). Days with patient visits in the upper quartile were identified based on an upper quartile limit of over 177 patient visits, and days with patient visits in the lower quartile were identified based on a limit of less than 153 patient visits. Proportion of high acuity patients (ESI 2 or 1) was calculated by the sum of patients with ESI of 1 or 2 divided by the total number of daily visits. Weighted mean ESI was calculated by multiplying number of patients per ESI category by ESI number and dividing by total patient visits. Total patient visits for the previous day was calculated based on the previous day's total visits and used as a proxy measurement of patient backlog in the ED that might affect department efficiency.

All data cleaning and derived variable calculations were performed by primary author JF and author JF takes responsibility for the integrity of this data.

\section{Data Analysis}

Using calculated daily variables, we then compared performance on key measures for a period of one year before and after implementation of e-documentation to identify differences in performance following implementation. All statistical analysis and modeling was performed in freely available statistical software package R (r-project.org) (24) and conducted by primary author (JF) with assistance as needed from co-author ST. In all relevant calculations, a value of $\mathrm{p}$ $<0.05$ was used for the pre-specified threshold of statistical significance using two-tailed $t$ tests. 
The pre-specified primary outcome of this study was mean length-of-stay (LOS). The secondary outcomes included mean length-of-stay for admitted (LOSa) and discharged patients (LOSd), and mean time to disposition for admitted patients (TTD).

We first compared data from pre- and post-implementation periods across a variety of operational characteristics including: registered visits, mean daily visits, mean age, proportion of pediatric and geriatric patients, proportion of female patients, count and proportion of patients per ESI category, mean ESI, count and proportion of high acuity patients (ESI 1 or 2), mode of arrival, disposition, TTD, mean daily boarding hours, mean boarding hours per admitted patient, LOS, LOSa, and LOSd. These statistics were computed for both 8-week pre- and postimplementation periods and one-year pre- and post-implementation periods.

In addition, we examined the full data set in discrete blocks of 8 weeks in order to identify salient trends and anomalies across several key operational statistics, including: total registered visits, admission rate, observation admission rate, discharge rate, proportion of patients with ESI of 2 or 1, mean time to disposition for admitted patients, boarding hours per admitted patient, and mean LOS (all patients, admitted patients and discharged patients). These results are presented as an appendix to this report.

We then used descriptive statistics and multiple regression modeling to identify significant differences in our primary and secondary outcomes. We created a pre-specified multiple regression models for the primary outcome (LOS) and three secondary outcomes (LOSa, LOSd and TTD). Our pre-specified model incorporated the following variables: pre- or post-implementation (0 or 1), month of the year, day of the week, registered daily visits, registered daily visits from the preceding day, mean patient age, proportion of female patients, proportion of admissions, OBS admissions and discharges, proportion of patients with ESI $>2$, proportion of patients arriving by ambulance and total daily boarding hours. These variables were selected from all available departmental data based on their potential impact on departmental efficiency due to i) seasonal variation, ii) daily variation in patient demographics, volume and acuity, and iii) variation in hospital admissions and census (with boarding hours serving as a proxy measure of hospital census). The pre-specified variables incorporated in the model were selected by consensus of the authors prior to analysis of the dataset. We then applied this model for each of the outcome variables over a one-year pre- and post-implementation 
period and report an adjusted LOS (total, admitted and discharged) and time to disposition as compared to the unadjusted values across the two periods.

In addition, we applied this model for each of the outcome variables over an 8-week preand post-implementation period in order to capture short-term effects of eDoc implementation. We applied the same pre-specified model to compare an 8-week period immediately prior to implementation from $1 / 21 / 2013$ to $3 / 17 / 2013$ to the immediate post-implementation period from $3 / 18 / 2012$ to $5 / 12 / 2012$. We present the adjusted values for the primary and secondary outcome variables as compared to the unadjusted values for these periods.

Finally, we performed coarsened exact matching (CEM) for similar days across the preand post-implementation periods as a sensitivity analysis (25). Coarsened exact matching is a statistical method designed by Iacus, King and Porro (2011) for controlling for the confounding influence of pretreatment control variables in an observational dataset (an alternative to multiple regression modeling) (25). We used a matching algorithm from the CEM package designed for $\mathrm{R}$ statistical software to perform this analysis (26) (http://gking.harvard.edu/files/gking/files/cem.pdf). We utilized CEM to identify similar days based on pre-specified variables of month, day, registered visits, visits from the previous day, daily admission rate, proportion of patients with ESI 2 or 1, and total boarding hours. Matching was performed based on month of the year, day of the week and quartile of the remaining variables (visits, visits the previous day, admission rate, proportion of high acuity patients and boarding hours). Linear regression modeling was then used to compare these matched samples and estimate the "sample average treatment effect on the treated" (SATT). Due to an insufficient number of data points for matching in the 8-week pre and post-implementation sample, CEM analysis was performed only for one-year pre- and post-implementation periods.

\section{Study Approval}

This study was reviewed and approved by the Partners Health Care Institutional Review Board (IRB). The IRB application was prepared in its entirety by primary author JF and was reviewed and approved by all co-authors. This study does not involve any direct patient or physician contact, intervention or changes in patient care and thus the risk to safety of patients or staff was negligible. Waiver of consent was requested and the application was approved by the IRB. All data that included patient identifiers was kept on a password protected shared-file area 
maintained on secure internal hospital computer systems. This data was accessed and viewed only by study staff members and all patient identifiers were removed after the daily dataset was compiled. All data is presented in this report in aggregate form without any patient identifiers or protected healthcare information.

\section{Results}

We obtained available operational statistics for all patient encounters during the two-year study period. There were a total of 120,207 patient encounters during the designated study of March 18, 2012 to March 17, 2014. There were 60,870 total patient encounters during the preimplementation period and 59,337 total patient encounters during the post-implementation period. Patient populations during the pre- and post-implementation periods were similar; descriptive statistics for the one-year pre and post-implementation periods are shown in Table 1; descriptive statistics for 8-week periods before and after implementation are shown in Table 2. The complete study period was also divided into 8-week blocks in order to identify salient trends; this data is presented as Appendix A.

During the pre-implementation period, TTD for admitted patients was 3.00 hours, mean LOS was 4.29 hours for all patients, 6.47 hours for admitted patients and 3.49 hours for discharged patients. During the post-implementation period, mean time to disposition was 3.03 hours, the mean daily LOS was 4.43 hours for all patients, 6.66 hours for admitted patients and 3.52 hours for discharged patients. The net change for each of the primary and secondary outcomes is shown in Table 3-1.

We also examined 8-week periods before and after implementation in order to detect short-term changes in primary and secondary outcome variables. During the baseline 8-week period prior to implementation TTD was 3.06 hours, LOS was 4.16, LOSa was 5.97 and LOSd was 3.46. During the 8-week period after implementation these values were 2.91 hours, 3.87 hours, 5.22 hours and 3.36 hours respectively. The short-term change for each of the primary and secondary outcomes is shown in Table 3-2.

The pre-specified regression model was applied for the primary and secondary outcome variables for the full study period based on daily calculated variables (i.e. 365 data points for each of the pre- and post-implementation periods). The model was also applied to an eight-week pre- and post-implementation periods (56 data points for each of the pre- and post- 
implementation periods). The results of this analysis are shown on the righthand side of Tables 3-1 and 3-2 with adjusted values compared to unadjusted values for these periods. With application of the regression model there were no significant changes in the primary and secondary outcome variables for the 8-week post-implementation period although there was an overall trend towards decreasing values for all outcome variables. For the one-year study period overall, there was a significant change of $\Delta+0.10$ hours for overall LOS ad $\Delta+0.08$ hours for LOSd only.

Finally, coarsened exact matching analysis yielded matched samples of 102 data points from pre-implementation period and 106 data points from the post-implementation period. 263 data points in the pre-implementation period and 259 in the post-implementation period were pruned due to lack of an adequate match. When comparing the sets of similar days between the pre- and post-implementation periods, there was a significant change in LOS of +0.15 hours and LOSd of +0.17 hours. Complete results of this analysis are shown on the right-hand side of

Table 3-1. CEM was not performed for the 8-week short-term study period due to an inadequate sample size.

\section{Discussion}

Documentation or "charting" of clinical encounters imposes a significant time burden on ED staff - including attending physicians, residents, mid-level providers and nurses - and thus is a critical aspect of ED flow. Poor or inefficient documentation systems - both manual and electronic - have the potential to detrimentally impact departmental efficiency. Traditionally in the ED, as in other areas of medicine, patient encounters were recorded by handwritten notes or documented by hand using templates (e.g. T sheets). However, with recent national legislation encouraging the use of EHRs, provider groups and hospitals have begun rapidly transitioning to electronic systems in order to receive incentive payments and avoid penalties. This seismic shift in the healthcare arena represents both an opportunity for potential improvement in care delivery and a substantial hurdle to overcome due to new regulatory and infrastructure requirements and the financial implications of these changes.

The implementation of EHR systems holds the promise of improving healthcare efficiency, streamlining clinician workflow and reducing waste in the system. However, electronic systems do not guarantee such improvements and can themselves contribute to inefficiency and waste if not carefully designed, tested and deployed. Previously research has 
demonstrated that implementation of an EHR may lead to a transient or sustained increase in patient length of stay and decreased performance on other operational measures $(17,19,20,23)$; however, this effect has not been consistent across all studies $(16,21)$. Due to the potential effects on departmental efficiency in the setting of implementing new systems, it is important to fully characterize the impact of such changes in order to adequately plan for transition periods in the ED.

In this study, we examined the effect of transitioning our high-volume ED from paperbased documentation to an electronic charting system. Given that our institution had already implemented CPOE, we sought to characterize the isolated effect of e-documentation on departmental efficiency. Our hypothesis was that ED efficiency - as measured by the primary outcome of length of stay and the secondary outcomes of LOS for admitted and discharged patients and TTD - could be negatively affected in the short-term by the implementation of edocumentation but that this effect would not be sustained and the long-term effect would be neutral or positive. We performed a retrospective analysis of data for all patient visits over a oneyear study period before and after implementation of our home-grown e-documentation system.

Our analysis demonstrated a statistically significant increase in overall length-of-stay and length-of stay for discharged patients over the full study period. Based on linear regression results, this represents an increase of approximately 6 minutes per patient encounter and 5 minutes per discharged patient; coarsened exact matching yielded a statistically significant change in these categories equivalent to an additional 9 minutes for all patients and $\sim 10$ minutes for discharged patients. In comparing the one-year pre- and post-implementation periods there was an overall trend towards an increase in all outcomes. Over the short-term 8-week baseline and post-implementation periods there was an overall trend towards a decrease in all outcome variables but none of these differences was statistically significant after controlling for variations in operational statistics.

The results of our study indicate that implementation of e-documentation alone may increase patient length-of-stay by a consistent and statistically significant interval that has the potential to impact ED efficiency. This is consistent with what we have observed anecdotally in or department; ED staff report feeling that e-documentation tends to be more time-consuming than previous paper charting. Although the magnitude of this difference was small as measured in our study, even a difference of a few minutes could have a potential impact in a high 
throughput emergency department. For example, an additional 6 minutes per patient encounter may seem relatively small in magnitude; however, if this difference is solely attributable to additional time spent charting, then the change adds over 16 hours of charting time per day across the entire department serving approximately 165 patients per day. Significant changes to physician workflow, such as migrating to an electronic charting system, thus have the potential to create a large ripple effect in a high volume ED.

Based on our analysis, it appears that the overall difference in LOS is largely attributable to an increase in LOS for patients who were ultimately discharged. We did not identify a significant change in LOS for admitted patients. Admitting patients to the hospital is a multistep process and length of stay in the ED for admitted patients may be substantially extended by variables not captured in our sample such as hospital bed availability and specialty consults. Thus, it seems reasonable that a small increase in charting time may be not be apparent when examining LOS for admitted patients or detectable over a shorter time period (i.e. 8 weeks). In contrast, a difference might be more readily observable in discharged patients, who experience short LOS and whose discharge could be held up by additional charting time, or over a longer period where subtle differences in LOS accumulate.

Of note, we observed a non-significant trend towards shorter LOS and TTD over the immediate post-implementation period ( 8 weeks) that was most pronounced among admitted patients. This may simply represent an anomalous short-term change in operational characteristics that are not adequately controlled for in our model. Indeed, there was a substantial drop in boarding hours between these two periods, which likely reflects underlying changes to hospital census and volume that may have contributed to changes in the outcome variables outside of our model. Much of the short-term change in the outcome variables disappeared once boarding hours and other confounding variables were controlled for with our model.

On the whole, this study suggests that e-documentation may result in increases in lengthof-stay, with this effect coming primarily from added time for discharged patients $(\sim 5$ minutes/patient encounter). Some previous research has demonstrated that implementation of electronic health records (EHRs) may increase ED length-of-stay (17, 19. 20, 23). However, in another recent study of 23 EDs, no significant increase in LOS was observed after implementation of EHRs (21). Our results isolate the effect implementing electronic documentation from that of other EHRs functions (such as computerized provider order entry) 
and demonstrate a consistent and statistically significant increase in LOS at our single, highvolume, urban ED. These findings are consistent with previous research demonstrating an increase after EHR implementation and suggest that e-documentation is a contributing factor in this effect. Additional research will be needed to determine the generalizability of these results across difference e-documentation systems and EDs and to investigate ways to mitigate these impacts, such as using medical scribes and additional use of voice recognition.

\section{Limitations}

There are several potential limitations of this study. First, this represents a retrospective analysis of previously collected data and thus is limited in its ability to characterize significant differences compared to a controlled trial. However, it is not typically feasible to implement such a system within the constraints of a randomized, controlled trial and thus this pre- and postimplementation analysis serves as a reasonable surrogate.

In addition, due to the retrospective nature of this study, we were also limited to analysis of the operational variables typically collected by the BWH ED. It was not possible to expand our analysis beyond the variables mentioned above, which may have limited our ability to fully characterize the effects of implementation. A specific limitation of the retrospective data was that patient satisfaction data was only available on a monthly basis and only for patients discharged from the emergency department. As a result of this, we were unable to characterize any effect of eDoc implementation on patient satisfaction. However, monthly values were largely stable across the entire study period and it is thus unlikely that eDoc had a meaningful effect on satisfaction that was not captured.

In addition, this study also did not capture time that staff may have spent outside their shift hours to complete provider documentation using eDoc. Anecdotally, we know that many staff reported staying long hours to complete their documentation after the transition to eDoc. However, this would not have been detectable in this analysis of patient length of stay. Thus, our results may underestimate the impact of eDoc on departmental efficiency.

Given that eDoc is a custom developed electronic documentation system for our ED and HER, the generalizability of these results is also potentially limited. While the design of our documentation system is similar to other electronic documentation tools available, our results may not be applicable to other EDs. Relatively small differences in e-documentation workflow have the potential to substantially impact per-patient documentation time. 
Finally, this study represents a quantitative analysis of the impact of implementation on major quality and operational measures. Thus, it does not capture potentially important information such as physician buy-in, perceived system usability, or technical challenges in implementation. Evaluation of the implementation process using qualitative methods may also be valuable in the future.

\section{Conclusion}

The use of e-documentation in patient care is an important function enabled by the implementation of EHRs and is also a requirement for "meaningful use" under stage 2 of the federal EHR incentive program. The purpose of this study was to examine the impact of implementing e-documentation in the ED setting. In our single center study, using a customdeveloped system, implementation of e-documentation was associated with consistent and significant increases in overall length of stay and length of stay for discharged patients. This suggests that implementation of electronic provider documentation alone may have a moderate adverse affect on ED operational performance. Further study is warranted to better characterize this effect.

\section{Suggestions for Future Work}

Given that this study was a retrospective analysis of previously collected operational data, we were limited to the pre-specified variables collected by our emergency department. Length of stay for different populations and time to disposition were blunt measures of departmental efficiency and in examining these outcomes we may have overlooked more subtle findings regarding the impact of e-documentation on departmental efficiency. Several variables that could be potentially valuable in this study were not available to us, including patient ethnicity, daily emergency department satisfaction survey results, time spent by clinicians on charting during and after each shift, patient volume per physician and numerous others. A prospective analysis of implementation at a similar site could add significant additional insight into the effects of edocumentation. This would allow for the pre-specification of variables of interest and thus provide richer quantitative results.

Additionally, given that our study examined data from a single site and detected only a relatively small increase in length of stay, it would be of value to repeat this analysis across multiple sites in order to validate and improve the generalizability of our findings. Our findings 
may be system- or institution-specific and it would be useful to know if other hospitals have experienced larger increased in length of stay or decreases in length of stay using different edocumentation systems or due to differing operational characteristics of their EDs.

Importantly, this study also includes only quantitative operational data related to the implementation of e-documentation. As a result, our investigation does not capture potentially valuable qualitative information, such as physician buy-in, system usability, and the technical challenges around implementation. Frequently, factors such as these play a significant role in the success or failure of new EHR functionality. Qualitative methods, such as surveys, direct observation or clinician interviews, could be of great value in further characterizing the effects of implementing this new electronic documentation system. Such methods would be able to better characterize the challenges of implementation, the ways in which it altered clinician workflow, and the perceived impact of the new system (positive or negative) that cannot be divined from operational statistics. Qualitative data could add significant richness to the operational data described in this report and the prospective collection of qualitative data may be valuable to include as a component of future implementation projects.

Given that that the results of our study suggests that that e-documentation adds significantly to ED length of stay - it will also be critical in future studies to identify interventions to mitigate this impact. For example, future research could focus on working with EHR vendors to perform user-centered design and improve the usability of EHRs to speed documentation time. It would also be valuable to explore the potential impact of adjusting staffing, adding medical scribes, and using voice recognition software to mitigate the impact of electronic documentation on ED operations.

\section{Acknowledgements}

We would like to thank Hillary Cornish for her assistance with data collection for this study. We would also like to thank the Brigham and Women's Hospital Emergency Department Information Systems team for their hard work designing, developing, and implementing this electronic documentation module. The lead author (JF) would also like to thank co-authors AL and ST for their advising and assistance with analysis in the completion of this scholarly project. All authors have no conflicts-of-interest to disclose relevant to this report. 


\section{References}

1. Blumenthal D. Stimulating the adoption of health information technology. N Engl J Med. 2009 Apr 9;360(15):1477-9. doi: 10.1056/NEJMp0901592. Epub 2009 Mar 25.

2. DesRoches CM, Charles D, Furukawa MF, Joshi MS, Kralovec P, Mostashari F, Worzala C, Jha AK. Adoption of electronic health records grows rapidly, but fewer than half of US hospitals had at least a basic system in 2012. Health Aff (Millwood). 2013 Aug;32(8):1478-85. doi: 10.1377/hlthaff.2013.0308. Epub 2013 Jul 9.

3. Wright A, Henkin S, Feblowitz J, McCoy AB, Bates DW, Sittig DF. Early Results of the Meaningful Use Program for Electronic Health Records. N Engl J Med 2013; 368:779-780, February 21, 2013. DOI: 10.1056/NEJMc1213481.

4. Adler-Milstein J, DesRoches CM, Furukawa MF, Worzala C, Charles D, Kralovec P, Stalley S, Jha AK. More than half of US hospitals have at least a basic EHR, but stage 2 criteria remain challenging for most. Health Aff (Millwood). 2014 Sep;33(9):1664-71. doi: 10.1377/hlthaff.2014.0453. Epub 2014 Aug 7.

5. Centers for Disease Control and Prevention Website. "Meaningful Use: Introduction." http://www.cdc.gov/ehrmeaningfuluse/introduction.html/. Accessed February 27, 2015.

6. Blumenthal D, Tavenner M. The "Meaningful Use" Regulation for Electronic Health Records. N Engl J Med 2010; 363:501-504 August 5, 2010 DOI: 10.1056/NEJMp1006114.

7. Centers for Medicare \& Medicaid Services Website. CMS.gov. "EHR Incentive Program:

Registration \& Attestation." http://www.cms.gov/Regulations-and-

Guidance/Legislation/EHRIncentivePrograms/RegistrationandAttestation.html/. Accessed

February 27, 2015.

8. Centers for Medicare \& Medicaid Services Website. CMS.gov. "EHR Incentive Program: Stage 2 Attestation User Guide For Eligible Hospital and Critical Access Hospital." http://www.cms.gov/Regulations-andGuidance/Legislation/EHRIncentivePrograms/Downloads/UserGuides_Stage2AttestationEHCA Hs.pdf. Accessed February 27, 2015.

9. Baumlin KM, Shapiro JS, Weiner C, Gottlieb B, Chawla N, Richardson LD. Clinical information system and process redesign improves emergency department efficiency. Jt Comm J Qual Patient Saf. 2010 Apr;36(4):179-85. PubMed PMID: 20402375.

10. Zikos D, Diomidous M, Mpletsa V. The Effect of an Electronic Documentation System on the Trauma Patient's Length of Stay in an Emergency Department. J Emerg Nurs. 2014 Jan 20. doi:pii: S0099-1767(13)00490-X. 10.1016/j.jen.2013.10.008.

11. Boger E. Electronic tracking board reduces ED patient length of stay at Indiana Hospital. $J$ Emerg Nurs. 2003 Feb;29(1):39-43.

12. Daniel GW, Ewen E, Willey VJ, Reese Iv CL, Shirazi F, Malone DC. Efficiency and economic benefits of a payer-based electronic health record in an emergency department. Acad Emerg Med. 2010 Aug;17(8):824-33. doi: 10.1111/j.1553-2712.2010.00816.x. 
13. Spalding SC, Mayer PH, Ginde AA, Lowenstein SR, Yaron M. Impact of computerized physician order entry on ED patient length of stay. Am J Emerg Med. 2011 Feb;29(2):207-11. doi: 10.1016/j.ajem.2009.10.007.

14. Landman AB, Bernstein SL, Hsiao AL, Desai RA. Emergency department information system adoption in the United States. Acad Emerg Med. 2010 May;17(5):536-44. doi: 10.1111/j.1553-2712.2010.00722.x.

15. Jamoom E, Hing E. Progress with electronic health record adoption among emergency and outpatient departments: United States, 2006-2011. NCHS data brief, no 187. Hyattsville, MD: National Center for Health Statistics. 2015.

16. Mathison $\mathrm{Dj}$, Chamberlain J. Evaluating the impact of the electronic health record on patient flow in a pediatric emergency department. Appl Clin Inform. 2011 Feb 2;2(1):39-49. doi: 10.4338/ACI-2010-08-RA-0046. Print 2011.

17. Park SY, Lee SY, Chen Y. The effects of EMR deployment on doctors' work practices: a qualitative study in the emergency department of a teaching hospital. Int J Med Inform. 2012 Mar;81(3):204-17. doi: 10.1016/j.ijmedinf.2011.12.001. Epub 2012 Jan 3.

18. Perry JJ, Sutherland J, Symington C, Dorland K, Mansour M, Stiell IG. Assessment of the impact on time to complete medical record using an electronic medical record versus a paper record on emergency department patients: a study. Emerg Med J. 2013 Aug 23. doi: 10.1136/emermed-2013-202479.

19. Spellman Kennebeck S, Timm N, Farrell MK, Spooner SA. Impact of electronic health record implementation on patient flow metrics in a pediatric emergency department. J Am Med Inform Assoc. 2012 May-Jun;19(3):443-7. doi: 10.1136/amiajnl-2011-000462. Epub 2011 Nov 3.

20. Ward MJ, Froehle CM, Hart KW, Collins SP, Lindsell CJ. Transient and sustained changes in operational performance, patient evaluation, and medication administration during electronic health record implementation in the emergency department. Ann Emerg Med. 2014 Mar;63(3):320-8. doi: 10.1016/j.annemergmed.2013.08.019. Epub 2013 Sep 14.

21. Ward MJ, Landman AB, Case K, Berthelot J, Pilgrim RL, Pines JM. The Effect of Electronic Health Record Implementation on Community Emergency Department Operational Measures of Performance. Ann Emerg Med. 2014 Jan 9. doi:pii: S0196-0644(13)01713-7.

10.1016/j.annemergmed.2013.12.019.

22. HealthIT.gov. "Benefits of Electronic Health Records (EHRs)."

http://www.healthit.gov/providers-professionals/benefits-electronic-health-records-ehrs/.

Accessed February 27, 2015.

23. Mohan MK, Bishop RO, Mallows JL. Effect of an electronic medical record information system on emergency department performance. Med J Aust. 2013 Mar 4;198(4):201-4.

24. R Core Team (2013). R: A language and environment for statistical computing. $R$ Foundation for Statistical Computing, Vienna, Austria. ISBN 3-900051-07-0, URL http://www.Rproject.org/. 
25. lacus, Stefano M, Gary King, and Giuseppe Porro. 2011. Causal Inference Without Balance Checking: Coarsened Exact Matching. Political Analysis. Copy at http://j.mp/iUUwyH.

26. Gary King, Personal Website. "CEM:Coarsened Exact Matching Software, CEM Package for R." http://www.gking.harvard.edu/cem/. Accessed February 27, 2015. 
Table 1: Pre- and post-implementation population characteristics

\begin{tabular}{|c|c|c|c|c|}
\hline \multirow{2}{*}{ Total Visits } & \multicolumn{2}{|c|}{$\begin{array}{l}\text { Pre-implementation } \\
(3 / 18 / 2012-3 / 17 / 2013)\end{array}$} & \multicolumn{2}{|c|}{$\begin{array}{l}\text { Post-implementation } \\
(3 / 18 / 2013-3 / 17 / 2014)\end{array}$} \\
\hline & 60,870 & & 59,337 & \\
\hline Visits (SD)* & $166.8(18.6)$ & & $162.6(18.3)$ & \\
\hline Age (SD) & $48.4(1.8)$ & & $48.7(1.7)$ & \\
\hline \multicolumn{5}{|l|}{ Sex } \\
\hline Female & 36,578 & $60.1 \%$ & 35,548 & $59.9 \%$ \\
\hline \multicolumn{5}{|l|}{ ESI } \\
\hline 1 & 833 & $1.4 \%$ & 929 & $1.6 \%$ \\
\hline 2 & 19,349 & $31.8 \%$ & 17,357 & $29.3 \%$ \\
\hline 3 & 30,344 & $49.9 \%$ & 30,607 & $51.6 \%$ \\
\hline 4 & 8,903 & $14.6 \%$ & 9,073 & $15.3 \%$ \\
\hline 5 & 1,428 & $2.3 \%$ & 1,329 & $2.2 \%$ \\
\hline High Acuity $(\geq 2)$ & 20,182 & $33.2 \%$ & 18,286 & $30.8 \%$ \\
\hline Mean ESI & 2.85 & & 2.87 & \\
\hline \multicolumn{5}{|l|}{ Mode of Arrival } \\
\hline Ambulance & 17,044 & $28.0 \%$ & 16,915 & $28.5 \%$ \\
\hline \multicolumn{5}{|l|}{ Disposition } \\
\hline Admit & 16,757 & $27.5 \%$ & 16,790 & $28.3 \%$ \\
\hline OBS & 6,410 & $10.5 \%$ & 6,007 & $10.1 \%$ \\
\hline Home & 35,583 & $58.5 \%$ & 34,543 & $58.2 \%$ \\
\hline Boarding Hours (SD) & $161.8(89.2)$ & & $169.8(100.6)$ & \\
\hline $\begin{array}{l}\text { Boarding Hours per } \\
\text { Admitted Patient }\end{array}$ & 3.52 & & 3.69 & \\
\hline Length of Stay (LOS) & $4.29 \mathrm{hrs}$ & & $4.43 \mathrm{hrs}$ & \\
\hline LOS (Admit) & $6.47 \mathrm{hrs}$ & & $6.66 \mathrm{hrs}$ & \\
\hline LOS (Discharge) & $3.49 \mathrm{hrs}$ & & $3.52 \mathrm{hrs}$ & \\
\hline $\begin{array}{l}\text { Time to Disposition per } \\
\text { Admitted Patient }\end{array}$ & $3.00 \mathrm{hrs}$ & & $3.03 \mathrm{hrs}$ & \\
\hline
\end{tabular}

* Computed statistics reflect daily counts or means unless otherwise noted 
Table 2: Short term differences, 8 week pre, 8 week post

\begin{tabular}{|l|l|l|l|l|}
\hline & \multicolumn{2}{|l|}{$\begin{array}{l}\text { Pre-implementation } \\
\mathbf{( 1 / 2 1 / 2 0 1 3 - 3 / 1 7 / 2 0 1 3 )}\end{array}$} & \multicolumn{2}{l|}{$\begin{array}{l}\text { Post-implementation } \\
\mathbf{( 3 / 1 8 / 2 0 1 3}-\mathbf{5} / \mathbf{1 2 0 1 3})\end{array}$} \\
\hline Total Visits & 8,708 & & 8,896 & \\
\hline Visits (SD) & $155.5(21.2)$ & & $158.9(17.0)$ & \\
\hline Age (SD) & $48.5(1.9)$ & & $48.2(1.8)$ & \\
\hline Sex & & & & \\
\hline Female & 5,272 & $60.5 \%$ & 5,333 & $59.9 \%$ \\
\hline ESI & & & & \\
\hline 1 & 172 & $2.0 \%$ & 164 & $1.8 \%$ \\
\hline 2 & 2,672 & $30.7 \%$ & 2,571 & $28.9 \%$ \\
\hline 3 & 4,285 & $49.2 \%$ & 4,547 & $51.1 \%$ \\
\hline 4 & 1,386 & $15.9 \%$ & 1,374 & $15.4 \%$ \\
\hline 5 & 190 & $2.2 \%$ & 231 & $2.6 \%$ \\
\hline High Acuity ( $\geq 2)$ & 2,844 & $32.7 \%$ & 2,735 & $30.7 \%$ \\
\hline Mean ESI & 2.83 & & 2.87 & \\
\hline Mode of Arrival & & & & \\
\hline Ambulance & 2,580 & $29.6 \%$ & 2,596 & $29.2 \%$ \\
\hline Disposition & & & & \\
\hline Admit & 2,441 & $28.0 \%$ & 2,367 & $26.6 \%$ \\
\hline OBS & 924 & $10.6 \%$ & 918 & $10.3 \%$ \\
\hline Home & 5,105 & $58.6 \%$ & 5,350 & $60.1 \%$ \\
\hline Boarding Hours (SD) & $125.9(52.1)$ & & $99.8(36.2)$ & \\
\hline $\begin{array}{l}\text { Boarding Hours per } \\
\text { Admitted Patient }\end{array}$ & 2.89 & & 2.36 & \\
\hline Length of Stay (LOS) & $4.16 \mathrm{hrs}$ & & $3.87 \mathrm{hrs}$ & \\
\hline LOS (Admit) & $5.97 \mathrm{hrs}$ & & $5.22 \mathrm{hrs}$ & \\
\hline LOS (Discharge) & $3.46 \mathrm{hrs}$ & & $3.36 \mathrm{hrs}$ & \\
\hline $\begin{array}{l}\text { Time to Disposition per } \\
\text { Admitted Patient }\end{array}$ & $3.06 \mathrm{hrs}$ & & $2.91 \mathrm{hrs}$ & \\
\hline & & & & \\
\hline
\end{tabular}


Table 3-1: Adjusted and unadjusted changes in outcome variables, full study period

\begin{tabular}{|l|c|c|c|c|c|}
\hline & \multicolumn{5}{|c|}{ Change in Primary \& Secondary Outcomes } \\
\hline & $\begin{array}{c}\text { Pre } \\
\text { (hours) }\end{array}$ & $\begin{array}{c}\text { Post } \\
\text { (hours) }\end{array}$ & $\Delta$ & $\begin{array}{c}\text { Adjusted } \\
\Delta\end{array}$ & CEM $\dagger$ \\
\hline Mean daily LOS* & \multicolumn{5}{|c|}{} \\
\hline All Patients & 4.29 & 4.43 & +0.14 & $+0.10^{*}$ & $+0.15^{*}$ \\
\hline Admitted Patients & 6.47 & 6.66 & +0.19 & +0.02 & +0.12 \\
\hline Discharged Patients & 3.49 & 3.52 & +0.03 & $+0.08^{*}$ & $+0.17^{*}$ \\
\hline Time to Dispo (Admitted Pts) & 3.00 & 3.03 & +0.03 & +0.05 & +0.09 \\
\hline
\end{tabular}

*Reaches $\mathrm{p}$ value for statistical significance $(\mathrm{p}<0.05)$

$\uparrow$ Coarsened exact matching, based on matched data from pre $(n=102)$ and post periods $(n=$ 106) by month, day and quartile of visits, visits the previous day, admit percentage, high acuity patients and boarding hours, unmatched data was excluded

\section{Table 3-2: Adjusted and unadjusted changes in outcome variables, 8 week baseline and post-implementation}

\begin{tabular}{|l|c|c|c|c|}
\hline & \multicolumn{4}{|c|}{ Change in Primary \& Secondary Outcomes } \\
\hline & Pre & Post & $\Delta$ & $\begin{array}{c}\text { Adjusted } \\
\text { (hours) }\end{array}$ \\
\hline Mean daily LOS & \multicolumn{4}{|l|}{} \\
\hline All Patients & 4.16 & 3.87 & -0.29 & -0.06 \\
\hline Admitted Patients & 5.97 & 5.22 & -0.75 & -0.19 \\
\hline Discharged Patients & 3.46 & 3.36 & -0.10 & -0.05 \\
\hline Time to Dispo (Admitted Pts) & 3.06 & 2.91 & -0.15 & -0.10 \\
\hline
\end{tabular}

*Reaches $\mathrm{p}$ value for statistical significance $(\mathrm{p}<0.05)$ 
N

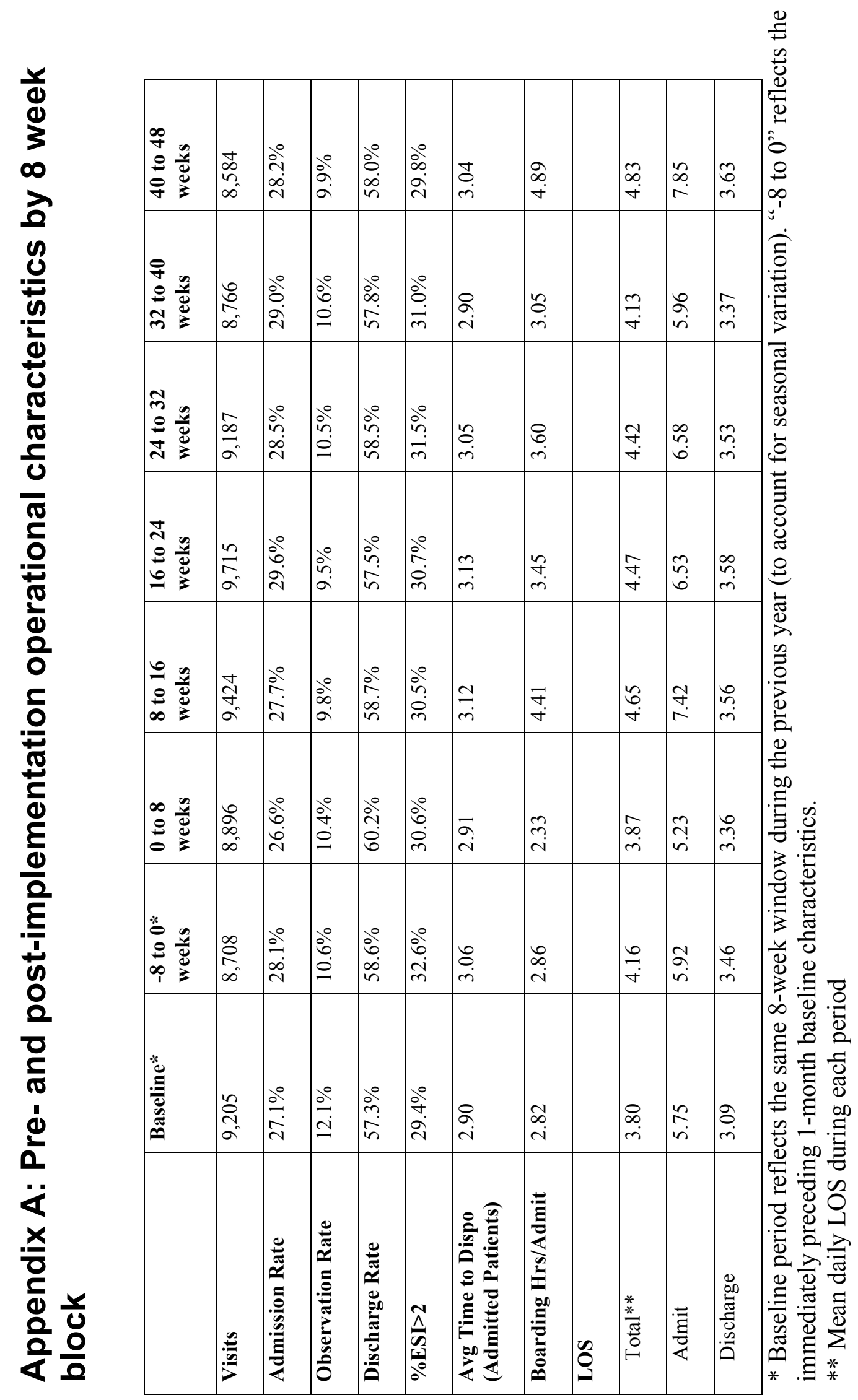


Figure 1: Primary and secondary outcome variables (LOS, LOSa, LOSd and TTD) over time by weekly average - One year before and after implementation of eDoc

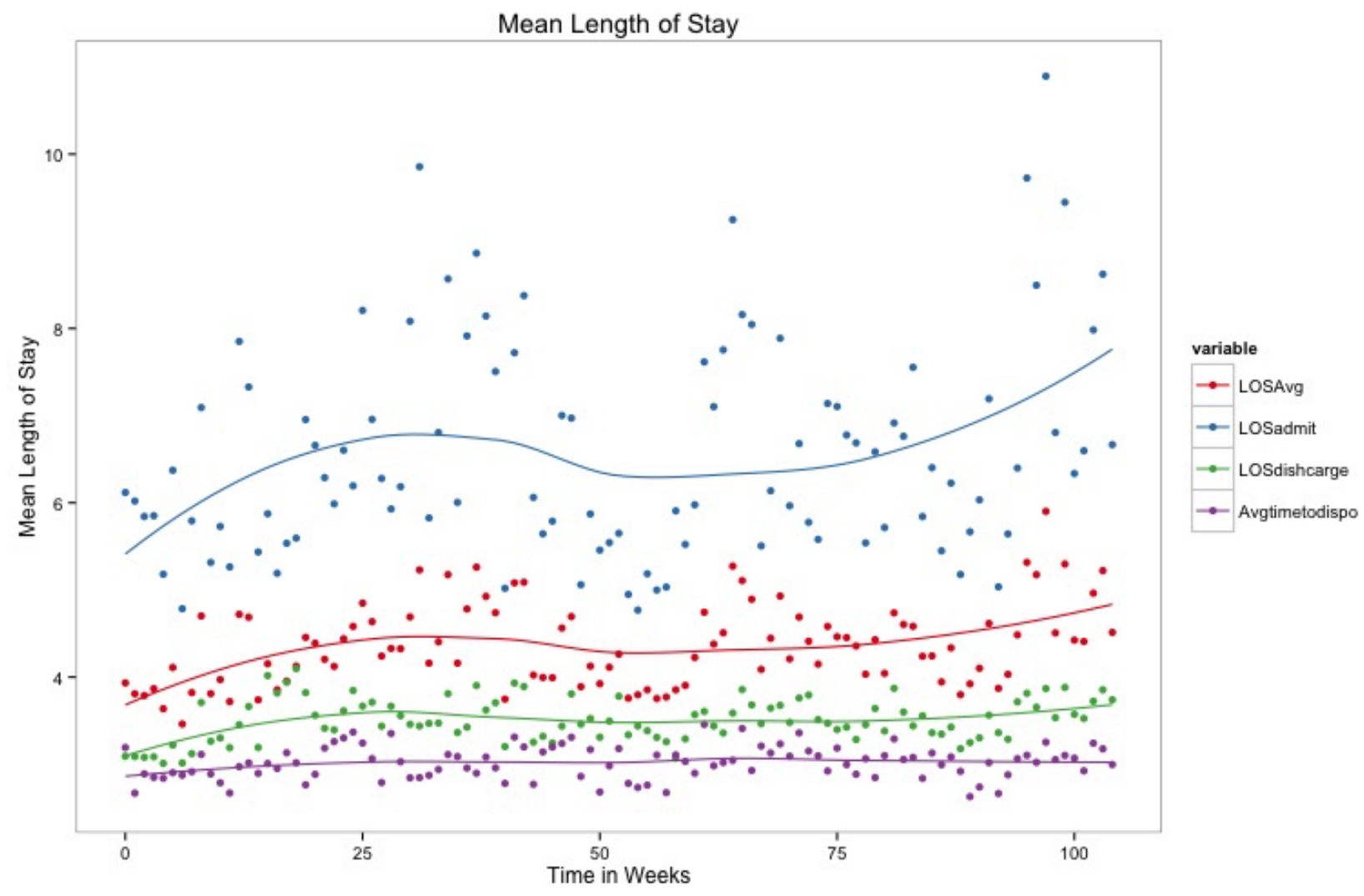


Figure 2: Primary and secondary outcome variables (LOS, LOSa, LOSd and TTD) over time by week - Eight weeks before and after implementation of eDoc

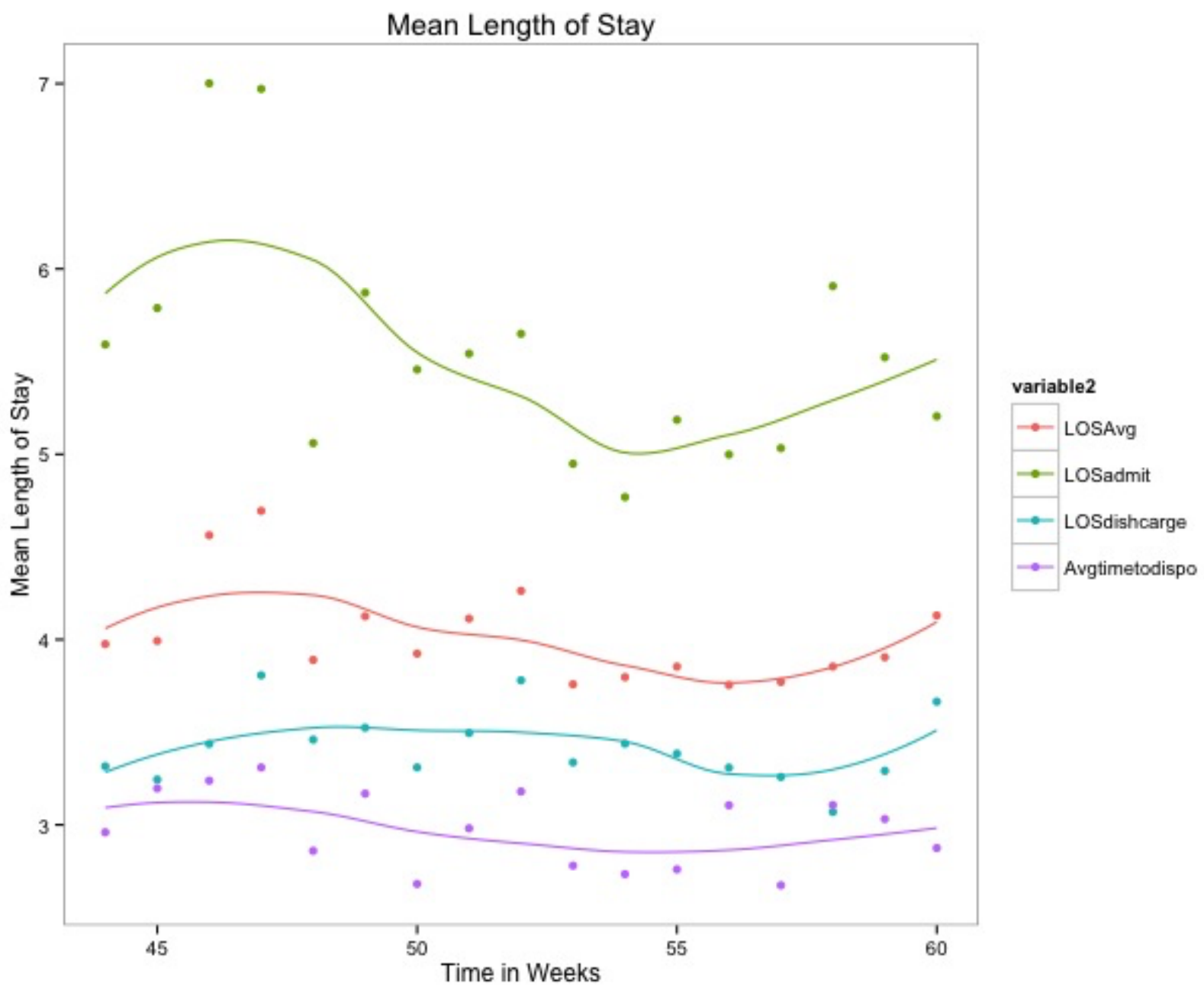

